Published in : Neuroscience (2006), 139 (1), 209-221

Status : Postprint (Author's version)

\title{
Exploration of the neural substrates of executive functioning by functional neuroimaging
}

\author{
Fabienne Collette $^{1,2}$, Michaël Hogge ${ }^{1}$, Eric Salmon ${ }^{2,3}$, Martial Van der Linden ${ }^{4}$ \\ ${ }^{1}$ Department of Neuropsychology, University of Liège, Belgium \\ ${ }^{2}$ Cyclotron Research Centre, University of Liège, Belgium \\ ${ }^{3}$ Department of Neurology, University of Liège, Belgium \\ ${ }^{4}$ Cognitive Psychopathology Unit, University of Liège, Belgium and University of Geneva, Switzerland
}

\begin{abstract}
This review presents neuroimaging studies that have explored the cerebral substrates of executive functioning. These studies have demonstrated that different executive functions not only recruit various frontal areas but also depend upon posterior (mainly parietal) regions. These results are in accordance with the hypothesis that executive functioning relies on a distributed cerebral network that is not restricted to anterior cerebral areas. However, there exists an important heterogeneity in the cerebral areas associated with these different processes, and also between different tasks assessing the same process. Since these discrepant results could be due to the paradigms used (subtraction designs), recent results obtained with conjunction and interaction analyses are presented, which confirm the role of parietal areas in executive functioning and also demonstrate the existence of some specificity in the neural substrates of the executive processes of updating, shifting and inhibition. Finally, fMRI studies show that the activity in cerebral areas involved in executive tasks can be transient or sustained. Consequently, to better characterise the functional role of areas associated with executive functioning, it is important to take into account not only the localisation of cerebral activity but also the temporal pattern of this activity.
\end{abstract}

Keywords: Executive functions, Functional imaging, PET, fMRI

Corresponding author: Fabienne Collette, Neuropsychology Unit, Boulevard du Rectorat 3 (B33), B-4000

Liege (Sart Tilman), BELGIUM, TEL: +32 436622 74, FAX: +32 436628 75, E-MAIL:

f.collette@ulg.ac.be

Acknowledgements

F. Collette is Research Associate and M. Hogge is Research Fellow at the National Fund for Scientific Research (FNRS) of Belgium. 
Published in : Neuroscience (2006), 139 (1), 209-221

Status : Postprint (Author's version)

\section{Introduction}

Executive functioning encompasses a series of high-level processes, the main function of which is to facilitate adaptation to new or complex situations, when highly practiced cognitive abilities or behavior no longer suffice. Indeed, even though most of our daily tasks can be performed in a routine way, some situations require the intervention of control mechanisms to produce appropriate and efficient performance. A great number of separate functions have been attributed to control (or executive) processes, such as inhibition of prepotent responses, initiation of behaviour, planning of action, hypothesis generation, cognitive flexibility, judgement and decision making, and feedback management.

The identification of the cognitive processes that can be attributed to executive functioning comes from various domains. Thus, single-case analyses of brain-damaged patients demonstrated the existence of double dissociations between tasks assessing different executive functions, which supports the hypothesis that independent functions exist (Burgess \& Shallice, 1994; Shallice \& Burgess, 1991). For example, Shallice and Burgess (Burgess \& Shallice, 1994; Shallice \& Burgess, 1993) described frontal patients who performed well on a task assessing inhibitory processes (the Hayling task) but whose performance on another task evaluating rules detection was impaired (the Brixton test); other patients demonstrated the reverse profile. Another line of evidence for the existence of several distinct executive functions comes from group studies which examined these processes in several target populations, including normal young adults (Letho, 1996), normal elderly adults (Lowe \& Rabbit, 1997; Robbins et al., 1998) and braindamaged patients (Burgess, 1997; Burgess et al., 1998; Duncan et al., 1997). All these studies employed a large battery of widely used executive tasks ${ }^{1}$ and examined how well these tasks correlated with one another. One highly consistent pattern of results across these studies is that the intercorrelations among the different executive tasks are low and are often not statistically significant. Thus, the results of these group studies are often used to argue that the functions of the central executive are not unitary and hence need to be fractionated. In that context, Baddeley (1996) proposed, on the basis of a review of the literature, that the central executive be separated into four different functions: the capacity to allocate resources during the simultaneous execution of two tasks (dual-task coordination), the capacity to switch retrieval strategies (such as those used in a random generation task), the capacity to selectively attend to one stimulus and inhibit the disrupting effect of others, and the capacity to hold and manipulate information stored in longterm memory.

Finally, Miyake, Friedman, Emerson, Witzki and Howerter (2000) used latent variable analysis to determine to what extent different executive functions can be considered to be unitary (in the sense that they are a reflection of the same underlying mechanism or ability). The principle of latent variable analysis is to extract statistically what is common among several tasks selected to tap into a putative cognitive function, and then to use that purer latent variable factor to examine how different cognitive functions relate to one another. Thus, the authors administered a set of tasks requiring executive processes to a large group of young participants in order to examine the separability of three often-postulated executive functions (shifting, updating and inhibition). Confirmatory factor analysis indicated that these three executive functions are moderately correlated with one another, but are clearly separable, thus indicating both unity and diversity of executive functions. Moreover, structural equation modelling suggests that the tasks often used in cognitive and neuropsychological studies to explore executive functioning are not completely homogeneous in the sense that the three executive functions isolated contribute differentially to performance on complex executive tasks. For example, performance on the Wisconsin Card Sorting Test was related most strongly to the shifting function, while performance on the Tower of Hanoi task depends on inhibition processes. The only complex executive task that did not relate clearly to the three target executive functions was the dual task (requiring the simultaneous performance of a spatial maze task and a verbal word generation task). This suggests the possibility that the

\footnotetext{
${ }^{1}$ Executive tasks are experimental or clinical neuropsychological measures of executive functions, measured either with paper and pencil tests, or computer based methods.
} 
Published in : Neuroscience (2006), 139 (1), 209-221

Status : Postprint (Author's version)

simultaneous coordination of multiple tasks is an ability that is somewhat distinct from the three executive functions explored in this study. According to Miyake et al, at least two explanations may be proposed to take into account the intercorrelations between the three target executive functions. One is that all the tasks used in the study require the maintenance of goal and context information in working memory. In other words, they require 'controlled attention' capacity, which is a domain-free attentional capacity to actively maintain or suppress working memory representations according to the objectives of the task (Engle et al., 1999). The other interpretation is that all three executive functions involve an inhibitory capacity, which is considered by certain authors as a basic unit of working memory or executive functioning (e.g., Dempster \& Corkill, 1999; Zacks et al., 1996). Taken as a whole, the results of this study suggest that executive functioning is characterised by both unity and diversity of processes. Indeed, the three target executive functions are clearly distinguishable even though these functions are not completely independent and do seem to share some underlying commonality. More recently, Friedman and Miyake (2004) used the same procedure to demonstrate that the executive process of inhibition was indeed composed of a series of subprocesses. More specifically, resistance to proactive interference was clearly dissociated from prepotent response inhibition and resistance to distractor interference. From a clinical viewpoint, the existence of dissociations between different executive processes, as well as the existence of distinct subprocesses within a same executive process, is consistent with the observation that brain-damaged patients can exhibit deficits in one executive process but not in others (e.g. Burgess \& Shallice, 1994; Shallice \& Burgess, 1991, 1993).

The neural substrates of executive functioning were originally assumed to be located in the frontal lobes, since patients with lesions in the anterior part of the brain frequently demonstrated impaired performance on a wide range of tasks assessing executive functioning such as planning abilities, inhibition processes and rules detection (e.g., Burgess \& Shallice, 1996a,b; Owen et al., 1990; Shallice, 1982), although a normal executive performance was also reported in some cases. For example, studies using tasks requiring similar cognitive processes (such as dual task coordination or verbal fluency) have demonstrated either preserved (for example Ahola et al., 1996; Baddeley et al., 1997) or impaired performance in frontal-lobe patients (Cowey \& Green, 1996; Perret, 1974). Moreover, executive deficits are found more frequently following diffuse (Cowey \& Green, 1996; Simkins-Bullock et al., 1994) than focalised frontal lesions (Andrès \& Van der Linden, 2001; Andrès \& Van der Linden, 1998; Vilkki et al., 1996). On the other hand, there is also evidence suggesting that patients with nonfrontal lesions can show executive deficits similar to frontal patients (e.g., Andrès \& Van der Linden, 2000; Mountain \& SnowWilliams, 1993). Taken as a whole, these data seem to indicate that the presence of frontal lesions does not necessarily involve executive dysfunction and that executive processes are not exclusively based upon a network of prefrontal regions.

In conclusion, the results of cognitive and neuropsychological studies have not completely resolved a number of important questions about the nature and cerebral substrates of executive processes (Burgess, 1997; Rabbit, 1997). A wide range of cognitive functions are included under the label 'executive' and the exact nature and relationships of these functions are not yet completely understood (see, however, Friedman \& Miyake, 2004; Miyake et al., 2000). A second problem is that measures for executive tasks are less pure than those for non-executive ones (in the sense that they may also make demands on a variety of other cognitive skills or functions), and consequently participants' performance on these tasks may be contaminated by their performance of the non-executive requirements of the task. In that context, functional neuroimaging techniques (PET and fMRI) appear complementary to the cognitive study of brain-damaged patients and normal subjects. These techniques should allow a more precise identification of the cerebral areas involved in specific executive tasks (since the relationships between brain activity and behaviour will be directly observed and not inferred from cognitive impairments in brain-damaged patients) and should also be useful in examining the unitary or non-unitary nature of the central executive. 
Published in : Neuroscience (2006), 139 (1), 209-221

Status : Postprint (Author's version)

\section{The exploration of the neural substrates of executive functioning with functional neuroimaging}

\subsection{Subtraction cognitive studies}

A large number of studies exploring the neural substrates of executive functioning in normal subjects used task-specific analyses (or a 'subtraction design paradigm'). In this paradigm, changes in cerebral activity are compared in two tasks: (1) when subjects perform the executive task ('experimental task'); (2) when they perform a task that is similar to the experimental one in terms of the perceptual, motor and memory processes, but that does not require any executive processes to intervene ('control task'). This comparison should allow one to identify the cerebral areas associated with executive processes involved in the experimental task.

In a first series of studies, the neural substrates of executive functioning were explored with cognitive tasks roughly similar to those used in the clinical examination of brain-damaged patients. Thus, these studies used the Wisconsin Card Sorting Test (WCST, Berman et al., 1995; Nahagama et al., 1996; Ragland et al., 1997), the Tower of London (Backer et al., 1996; Dhager et al., 1999; Morris et al., 1993), random generation tasks (Jahanshashi et al., 2000) and verbal fluency tasks (Frith et al., 1991; Paulesu et al., 1997; Phelps et al., 1997). The WCST was associated with bilateral increases in cerebral activity in the dorsolateral, inferior parietal and occipital regions and, to a lesser extent, in the frontopolar, orbital and medial regions, as well as in the temporal areas. The Tower of London was initially designed to assess the planning abilities of frontally brain-damaged patients (Shallice, 1982). When this task was compared to tasks requiring the same sensorimotor components, increases in cerebral activity were found in the left prefrontal dorsolateral cortex, superior frontal cortex, (pre)motor cortex, and anterior cingulate and the right frontopolar region (Backer et al., 1996; Dagher et al., 1999; Morris et al., 1993). Increases in activity were also found (but to a lesser extent) in posterior cerebral areas: the precuneus, the left inferior parietal cortex, the superior parietal cortex bilaterally, as well as the occipital regions bilaterally (Backer et al., 1996; Dagher et al., 1999). Finally, Newman et al. (2003) demonstrated with functional connectivity analyses that, among these regions, the right prefrontal area may be more involved in the generation of a plan, whereas the left prefrontal area may be more involved in its execution. In addition, the right superior parietal region is more involved in attention processes while the left homologue is seen as visuospatial workspace. The random number generation task assesses the ability to give up an overlearned habit (counting in order from 1 to 9 ) in order to develop less usual strategies to generate the numbers. Relative to a counting task, random number generation was associated with significant activation of the left dorsolateral prefrontal cortex, the anterior cingulate, the superior parietal cortex bilaterally, the right inferior frontal cortex, and the left and right cerebellar hemispheres (Jahanshashi et al., 2000). Verbal fluency was consistently associated with the left inferior frontal gyrus (BA 45), the anterior cingulate and, to a lesser extent, the dorsolateral prefrontal cortex (BA 9/46), the right parahippocampal gyrus, the inferior parietal, and the left middle and superior frontal gyrus (BA 8) (Frith et al., 1991; Phelps et al., 1997). Verbal fluency tasks require information to be acceded and manipulated in long-term memory.

The results of these studies demonstrated that many cerebral areas (including both anterior and posterior regions) were highlighted when activity in the control task was subtracted from that in the executive task, making it quite difficult to attribute a functional role to these regions (see, however, Newman et al., 2003). One explanation of this widespread activity might be that these tasks involve more than one executive process. For example, random number generation depends on updating and inhibitory processes (Miyake et al., 2000). Moreover, given the number of executive and non-executive processes involved in these tasks, the development of reference tasks that control for all these aspects is not easy. So, the hypothesis that some of the activations related to the executive process of interest are actually due to other executive functions or to high-level non-executive functions (such as attentional or memory processes) cannot be ruled out.

Consequently, studies were undertaken to explore executive functioning using cognitive tasks considered to involve only one specific executive process. In the following paragraphs, we will present functional neuroimaging studies in normal subjects which explored the neural substrates of updating, 
Published in : Neuroscience (2006), 139 (1), 209-221

Status : Postprint (Author's version)

shifting, inhibition and dual task coordination. The neural substrates of these executive processes are of interest because Miyake et al. (2000) demonstrated that the first three processes are clearly separable at a cognitive level, although they have some common features. Moreover, these authors consider dual-task coordination to be a fourth potentially distinct executive process. In that context, we are interested in determining whether the distinctions found at the cognitive level can also be demonstrated with neuroimaging techniques (for a more detailed presentation of these studies, see Collette \& Van der Linden, 2002).

\subsubsection{The neural substrates of updating}

The updating process (Miyake et al., 2000; Shimamura, 2000) consists in continuously modifying the contents of working memory based on newer incoming information. Memory updating is involved in many everyday activities, such as learning and organising recently acquired information. The updating process was explored using the running span task for verbal material (Salmon et al., 1996; Van der Linden et al., 1999) and n-back tasks for letters, spatial positions or non-verbal material (Braver et al., 1997; Jonides et al., 1997; Schumacher et al., 1996; Smith et al., 1996). In the running span task, participants are required to watch strings of consonants of unknown length, and then to remember a specific number of recent items in the serial order. In $n$-back tasks, items are sequentially presented and subjects have to decide whether a given item is similar to the one presented $n$ items previously. Performance on the $n$-back task was associated with cerebral activity not only in the prefrontal dorsolateral cortex (BA 9/46), the inferior frontal cortex (BA 44), and the anterior cingulate, but also in cerebral posterior areas, such as the superior and posterior parietal cortex (BA 40/7) (see, for example, Braver et al. 1997; Cohen et al., 1997; Jonides et al., 1997; Schumacher et al., 1996; Smith et al., 1996). Moreover, the letter version of this task is associated with left (or bilateral) activations whereas spatial or object versions tend to activate righthemisphere regions (Owen et al., 1998; Smith et al., 1996); and this network of structures increases its overall level of activation with increases in task load rather than recruiting new areas to the task (Jonides et al., 1997). We conducted two PET studies using the running span task to explore the cerebral areas associated with the updating process. In the first study (Salmon et al., 1996), a large cerebral network involving anterior (right mid-dorsal prefrontal, left middle frontal and right frontal pole) and posterior (right inferior parietal and angular gyru and the left supramarginal gyrus) cerebral areas was associated with the updating process. However, a high memory load (six items) and a recognition procedure were used that could have induced cognitive strategies other than serial encoding and recall, as described in the original running span task (Morris \& Jones, 1990; Pollack et al., 1959). So we re-examined the cerebral areas associated with updating using a running span task with a memory load of four items and a serial recall procedure (Van der Linden et al., 1999). This time, the most significant increase in rCBF occurred quite specifically in the left frontopolar cortex (BA 10). Activation spread to the left middle frontal cortex (BA 46) and was also observed in the right frontopolar cortex. Taken as a whole, results obtained in these studies vary depending on the exact material and procedure used but show that the updating process is associated not only with various prefrontal areas (dorsolateral, inferior and cingulate) but also with parietal areas (posterior and superior). Among these areas, Van der Linden et al. (1999) highlighted the predominant role of the frontopolar cortex, which is associated with the evaluation and selection of internally generated information (Christoff \& Gabrielli, 2000).

\subsubsection{The neural substrates of shifting}

Efficient reactions to environmental stimuli require rapid and frequent shifts between the different aspects of the stimuli to be processed and also between several cognitive operations. Consequently, shifting ability is considered to be an important aspect of executive control (Norman \& Shallice, 1986). This executive function has classically been studied using task-shifting paradigms, in which participants rapidly repeat the same task or alternate between different tasks. A consistent finding with these paradigms is that response latencies are longer when subjects have to perform a switched task than when they have to perform a repeated task, and this decrease is called the switch cost. Neuroimaging studies using the subtraction design paradigm have shown that the shifting process depends on prefrontal, parietal 
Published in : Neuroscience (2006), 139 (1), 209-221

Status : Postprint (Author's version)

and subcortical areas (Fink et al., 1997; Gurd et al., 2002; Rogers et al., 2000; Wilkinson et al., 2001). In a recent meta-analysis, Wager et al. (2004) demonstrated that seven separate regions were reliably activated across a series of studies of attention shifting of various types, providing evidence for a unitary set of mechanisms underlying shifting. These regions included both posterior (parietal and occipital) and anterior areas (including the dorsolateral prefrontal cortex and anterior insula). With regard to the functional role of these regions, the superior parietal cortex is generally considered as a supramodal area involved in task switching, since this region is found to be activated not only when visual or spatial components are involved in the task (Vandenberghe et al., 2001) but also when verbal information is processed (Gurd et al., 2002). Sohn et al. (2000) attributed activity of the superior and posterior parietal cortex to endogenous goal-directed preparation for a subtask, while the inferior parietal cortex is claimed to be responsible for stimulus-driven completion during a specific subtask (Corbetta \& Shulman, 2002).

\subsubsection{The neural substrates of inhibition}

Inhibition is the third executive process identified by Miyake et al. (2000). It is generally considered that inhibitory control constitutes an important executive function and executive dysfunction represents one of the most frequent consequences of brain damage (Baddeley, 1986; Norman \& Shallice, 1986). Neuroimaging studies exploring inhibition processes with perceptual, motor or semantic paradigms have demonstrated the involvement of various regions located in the cingulate, prefrontal, parietal and temporal areas (Bench et al., 1993; Bush et al., 1998; Chee et al., 2000; Collette et al., 2001; Garavan \& Stein, 1999; Georges et al., 1994; Larrue et al., 1994; Pardo et al., 1990; Taylor et al., 1997). However, at this time, the exact role of the regions associated with inhibitory processes is not fully understood. Moreover, these regions are quite heterogeneous. Recently, for example, Nelson et al. (2003) demonstrated a dissociation in the cerebral areas associated with performance on different inhibitory tasks: the inferior frontal gyrus is activated when a subject needs to resolve interference among potentially conflicting attributes of a stimulus, whereas the anterior cingulate cortex is involved when conflicting stimulus-response associations are presented. Moreover, even though inhibition is frequently associated with the right inferior frontal gyrus (Aron et al., 2004), activity was found in the left inferior frontal gyrus when subjects had to resolve interference in verbal working memory tasks (D'Esposito et al., 1999; Jonides et al., 1998). As mentioned above, several authors consider that the term 'inhibition' has been overextended and that this concept in fact refers to several different processes (Friedman \& Miyake, 2004; Nigg, 2000). Thus, the tasks used in these studies may differ in their exact inhibitory requirements and consequently the cerebral areas involved may differ as well.

\subsubsection{The neural substrates of dual-task coordination}

Finally, there is still debate concerning the neural substrates of dual-task coordination, namely the concurrent processing of two behavioural tasks requiring disparate sensory and cognitive processes. Indeed, data from some studies supports the hypothesis that the dorsolateral prefrontal cortex and anterior cingulate are involved in the allocation and coordination of attentional resources (D'Esposito et al., 1995; Herath et al., 2001). However, other studies have not demonstrated any additional prefrontal activity during dual-task performance (Adcock et al., 2000; Bunge et al., 2000; Klingberg, 1998; Smith et al., 2001). Overall, results of these studies seem to indicate that no specific cortical area is associated with any specific cognitive process for dual-task performance and that the simultaneous execution of two tasks depends mainly upon greater activity of (Adcock et al., 2000; Klingberg, 1998) or interaction between (Bunge et al., 2000; Smith et al., 2001) the cerebral areas already activated for the single tasks.

One possible explanation of these discrepancies may be the use of single tasks that already involved executive functioning, leading to activity in the frontal areas during single-task performance in most of these studies. Consequently, we recently explored dual-task management using simple verbal and auditory discrimination tasks that elicit no prefrontal activation (at the very lenient statistical threshold used) when performed in isolation (Collette et al., 2005a). In that way, we formally determined whether dual-task management involves additional activity at the level of the prefrontal cortex or greater activity only in the posterior cerebral areas already activated by the single tasks. The comparison of dual-task 
Published in : Neuroscience (2006), 139 (1), 209-221

Status : Postprint (Author's version)

management (namely, the simultaneous performance of auditory and visual discrimination tasks) to the single tasks (namely, the performance of the auditory and visual discrimination tasks in isolation) evidenced a left-sided fronto-parietal network. This network is composed of the inferior frontal sulcus (BA 9/46 and BA 44/45), anterior prefrontal cortex (BA 10/47), posterior middle frontal gyrus (BA 6) and left inferior parietal gyrus (BA 40). Increased activity was also observed in the cerebellum. With reference to the neuroimaging literature, the following roles can be proposed for these regions. Activity in the left inferior frontal sulcus, extending to the middle frontal gyrus (BA 9/46) has previously been correlated with dual-task management (D’Esposito et al., 1995). More generally, Koechlin et al. (2003) consider that BA 46 selects appropriate representations for action after the occurrence of behaviourally significant events. Another role recently attributed to that region is response selection, and more precisely motor processes that arise during the transition of perceptual mnemonic information into motor plans (Curtis \& D’Esposito, 2003; Pochon et al., 2001). The activation of the left posterior part of the inferior frontal sulcus may be associated with the coordinated manipulation of different stimuli, since activity in this region was also found in a task requiring subjects to construct an integrated representation of two stimuli presented simultaneously (Collette et al., 2005a). Anterior ventrolateral activation has been reported to subserve selection in relatively complex situations, an important process in dual tasks (Collette et al., 2001). With regard to left posterior middle frontal activation (BA 6), the role of this area has been interpreted in terms of rehearsal processes (Awh et al., 1996; Paulesu et al., 1993). The left inferior parietal gyrus (BA 40) may be involved in the attentional shifting necessary to simultaneously maintain activated auditory and visual information, since this regions was frequently associated with various switching tasks (Garavan et al., 2000; Kübler et al., 2003).

\subsubsection{Conclusions on cognitive subtraction studies}

In summary, studies exploring the neural substrates of executive functioning using cognitive subtraction designs have demonstrated that many cerebral areas are associated with the different executive processes. Heterogeneous regions were found to be activated, not only by the four executive processes reviewed here but also by a single process, depending on the exact requirements of the tasks administered. Taken as a whole, these studies clearly demonstrate that the different executive functions depend upon the intervention of both prefrontal and posterior (mainly parietal) regions during the performance of various executive tasks. This is in accordance with the hypothesis that executive functioning is based on a network of anterior and posterior cerebral areas and is not localised only within the frontal lobes (D'Esposito \& Grossman, 1996; Fuster, 1993; Morris, 1994; Weinberger, 1993).

More specifically, Duncan and Owen (2000) proposed that a specific frontal lobe network including the mid-dorsolateral, mid-ventrolateral and dorsal anterior cingulate cortex is consistently associated with a broad range of tasks requiring, among other processes, response selection, working memory maintenance and stimulus retrieval, while much of the remainder of the frontal cortex, including most of the medial and orbital regions, is largely insensitive to these task demands. In support of this hypothesis, Collette and Van der Linden (2002) showed that some prefrontal areas (BA 9/46, BA 10 and anterior cingulate gyrus) are systematically activated by a wide range of executive tasks, suggesting that they are involved in general executive processes. However, other frontal areas (BA 6, 8, 44, 45, 47) and parietal regions (BA 7 and BA 40) are also activated during the performance of executive tasks. Since these regions are involved less systematically in the different executive processes explored in that review, it was hypothesised that they have more specific functions. Finally, in a recent meta-analysis, Wager and Smith (2003) showed that different executive processes (continuous updating, memory for temporal order, manipulation of information in working memory and selective attention) are associated with specific cerebral areas. For example, manipulation of information (including dual-task requirements or mental operations of switching and inhibition) most frequently activates the right inferior prefrontal cortex (BA 10 and 47). The superior frontal cortex (BA 6, 8 and 9) responds most when working memory must be continuously updated and when memory for temporal order must be maintained. Selective attention to features of a stimulus to be stored in working memory activates the medial prefrontal cortex (BA 32) in storage tasks. The posterior parietal cortex (BA 7) is involved in all three of these executive processes and 
Published in : Neuroscience (2006), 139 (1), 209-221

Status : Postprint (Author's version)

is also associated with basic control over the focus of attention. These results show that executive functions may be fractionated into different component processes and that these components are associated with specific cerebral areas.

However, these neuroimaging studies using task-specific analyses (or cognitive subtraction design) suffer from some limitations. Indeed, the specificity of active vs. baseline differences for the executive function under study is often questionable (Friston et al., 1996; Sidtis et al., 1999). The multicompound aspect of executive tasks leads to major difficulties in finding experimental and control tasks that enable one to isolate one specific executive process. Another problem is that each executive function can be assessed by several cognitive tasks. For example, inhibition has been evaluated by the Stroop test (Stroop, 1935), the Hayling task (Burgess \& Shallice, 1996b), the stop-signal paradigm (Logan, 1994), the antisaccade task (Roberts, 1994), the negative priming (Tipper, 1991) and the directed forgetting paradigm (Zacks et al., 1996). Finally, as indicated previously, subjects' performance on executive tasks is also determined by their ability to process the non-executive aspects of the tasks. Consequently, the pattern of cerebral activity associated with the executive process of interest could be modulated by the non-executive requirements of the task. Arguments for this hypothesis come from the studies of Salmon et al. (1996) and Van der Linden et al. (1999), which demonstrated that the neural substrates of updating varied depending on the memory load and the procedure used (serial recall versus recognition).

\subsection{Conjunction analysis paradigms}

2.2.1 Conjunction tasks

The use of subtractive paradigms does not appear to be the most appropriate method of studying executive functioning. Moreover, up till now, functional neuroimaging studies have explored executive tasks in isolation, without taking into account the fact that the same process can be assessed by various tasks and that the non-executive requirements of a task modulate the cerebral areas involved in the running of the executive processes. In that context, we recently re-examined the neural substrates of the executive processes of updating, shifting and inhibition (Collette et al., 2005c). Each process was explored with three different tasks, and conjunction designs were used instead of substraction designs. The principle of conjunction design is the search for convergence between cerebral areas activated by different tasks. More specifically, in a conjunction analysis, a series of tasks having a cognitive process in common (and differing according to whether they use other processes) are administered, as well as control tasks matched to each experimental task. The analysis determines cerebral areas common to the multiple comparisons of matched experimental and control tasks. This approach allows for better control over the multi-compound aspect of executive tasks and the difficulty of finding control tasks that are matched for the 'non-interesting' cognitive processes. To determine the cerebral areas associated with these three executive processes, the tasks used by Miyake et al. (2000) were adapted to the PET methodology and matched control tasks were developed. The neural substrates common to various tasks assessing updating, shifting and inhibition were explored separately in three experiments. Moreover, a conjunction analysis was also performed on all executive tasks in order to identify the cerebral areas activated by all three executive functions. Interaction analyses were used to highlight the brain regions specifically associated with each executive process, whichever the exact task administered. Such an analysis determines cerebral areas that present a differential activation between two experimental and two matched control tasks.

The eight tasks administered were the following. ${ }^{2}$ The neural substrates of updating in working memory were explored using running span tasks for consonants, words and sounds. In the consonant updating task, lists of items were presented. Subjects were not informed of the length of the list before presentation and they were asked to rehearse only the last four items silently and to remember them in order. In the control task, only sequences of four items were presented. With regard to word updating, exemplars of different semantic categories were presented. In the control task, the participants memorised only the exemplars belonging to a specified semantic category, while in the updating task, participants had

\footnotetext{
${ }^{2}$ A stop-signal task was also included in the initial study by Miyake et al. (2000). However, this task was not found to be clearly associated with their inhibitory factor and consequently was not used in our study.
} 
Published in : Neuroscience (2006), 139 (1), 209-221

Status : Postprint (Author's version)

to retain only the last exemplar presented of each category and then recall them freely at the end of the series. Finally, sound updating consisted of the presentation of three kinds of sounds. In the control task, subjects had to detect each occurrence of a predetermined sequence of three sounds while the updating task involved detecting the fourth occurrence of each kind of tone.

With regard to the shifting function, the three experimental tasks required subjects to continuously alternate between two cognitive processes. The matched control tasks required them to perform each of the two processes of interest in isolation. In the first shifting task, subjects had to perform arithmetic operations, namely to alternately add and subtract three to each number presented. In the second shifting task, number-letter pairs were visually presented and subjects had to process either the number (odd/even judgement) or the letter (consonant/vowel judgement) according to the position of the pair on the screen. Finally, the third condition consisted of the presentation of Navon Figures (Navon, 1977) in which the lines of a large 'global' figure (e.g., a triangle) are composed of much smaller 'local figures (e.g., squares). In the shifting task, Navon figures with plain or dotted lines were randomly presented. Depending on the lines in which the figure was printed (plain or dotted), participants were instructed to say out loud the number of lines in the global, overall figure (plain) or the local, smaller figures (dotted line). Thus, when the lines of the stimuli changed across successive trials, the participants had to shift from examining the local features to the global features or vice versa. In the control task, only figures with one kind of line were presented and subjects did not have to shift from one level of processing to the other.

To explore inhibitory processes, the Stroop task (Stroop, 1935) and the antisaccade task (Roberts et al., 1994) were administered. In the experimental condition of the Stroop task, the words displayed represented a colour name and were written in a randomly selected mismatching colour (e.g., red written in green). Participants were instructed to verbally name the colour of each stimulus as fast as possible. In the control condition, randomised sequences of concrete words were displayed and subjects were instructed to read the items as quickly as possible. In the antisaccade task, a visual cue (black square) was presented at the farthest point to the left or right of the screen, followed by the presentation of the target stimulus (arrow) for 150 milliseconds. In the control tasks, the target stimulus was presented on the same side as the visual cue, while in the inhibition tasks it was presented on the opposite side. The participants' task was to indicate the direction of the arrow (left, up or right) with a key-press response. Given that the arrow appeared very briefly before being masked, participants were required to inhibit their reflexive response to the initial cue in the experimental task, because this response would make it difficult to correctly identify the position of the arrow.

With regard to statistical analyses, we were first interested in using conjunction analyses to determine the cerebral areas common to various tasks assessing the same executive process. We consider that areas evidenced in that way should be independent of the exact non-executive requirements of the tasks, and thus only associated with the executive process of interest. Next, a conjunction analysis on all tasks administered was performed in order to determine if there were any cerebral areas common to the three executive processes investigated. Finally, interaction analyses were also computed between the different executive processes, this time to show the specificity of each process in comparison to the two others.

The conjunction analysis on the updating tasks, compared to their respective control tasks, showed increased activity in the left frontopolar cortex (BA 10), in the left (BA 9) and right middle frontal gyrus (9/46) and bilaterally in the superior frontal sulcus (BA 6). Foci of cerebral activation were also found in the left inferior frontal gyrus (BA 44/45), the right lateral orbitofrontal area (BA 11), in the intraparietal sulcus bilaterally, in the right inferior parietal gyrus (BA 40) and in the cerebellum. A similar analysis applied to shifting tasks demonstrated the existence of significant foci of activation in the right supramarginal gyrus (BA 40), the left precuneus and the left superior parietal cortex (BA 7). Finally, when the two inhibitory tasks were jointly compared to their respective control tasks, no statistically significant foci of increased cerebral activity were found. However, a slight change in activity was detected in the right inferior frontal cortex, a region frequently considered to be associated with inhibitory processes (e.g., Aron et al., 2004). 
Published in : Neuroscience (2006), 139 (1), 209-221

Status : Postprint (Author's version)

We were also interested to see whether any cerebral areas are common to these three executive processes. So a conjunction analysis was performed to compare the eight experimental tasks to the eight control tasks. This analysis demonstrated that the common cerebral areas activated by the executive processes of updating, shifting and inhibition are posterior regions located in the left superior parietal gyrus (BA 7), the right intraparietal sulcus and, at a lower statistical threshold, in the left middle (BA 9 and BA 10/46) and inferior frontal gyri (BA 45).

Finally, interaction analyses demonstrated that updating was specifically associated with the right superior frontal sulcus (BA 6), the left frontopolar cortex (BA 10) and the right inferior frontal sulcus (BA 10) (in comparison to shifting), and with the left intraparietal sulcus and frontopolar gyrus (in comparison to inhibition). Cerebral areas specifically associated with the shifting process were found only in the comparison with inhibition. These areas are located in the left intraparietal sulcus. Finally, when inhibition processes were contrasted to updating, they were found to be associated with activation in the right orbitofrontal gyrus (BA 11). The comparison of inhibition and shifting demonstrated increased activity in the right middle/superior frontal gyrus (BA 10) for inhibition.

Taken as a whole, these results demonstrated (1) that different tasks assessing the same executive process (as evidenced at the cognitive level) are associated with common cerebral areas; and (2) that the three executive processes explored in this study are in fact subserved by different cerebral areas, but that certain cerebral areas are involved no matter what executive process is performed. These neuroimaging data provide some important information concerning the unity and diversity of executive functioning. The unity is attested by the existence of cerebral areas commonly involved in the running of several different executive processes. On the other hand, the description of areas activated by one of the processes, but not the other two, is in agreement with the hypothesis that there is some degree of specificity in executive functioning.

The results obtained with conjunction analyses on tasks assessing a single executive process and with interaction analyses between the three processes are consistent with those of previous cognitive subtraction studies. With regard to updating, earlier studies had also demonstrated the involvement of a large antero-posterior cerebral network (for a review, see Collette \& Van der Linden, 2002). Given the large number of regions commonly associated with the three updating tasks, we may suppose that various high-level processes intervene during the performance of these tasks. In particular, the updating function depends on a series of processes such as shifting from internal to external information, discarding irrelevant items, tracking serial order, and repositioning items. More specifically, the frontopolar cortex had previously been found to be associated with the evaluation and selection of internally generated information (Christoff \& Gabrielli, 2000). This is a key process for updating, since subjects must continuously compare new information with items that have already been encoded so that they can keep only a specific set of the last items presented in working memory. The shifting function is mainly associated with posterior parietal areas (right supramarginal gyrus, left precuneus, left superior parietal gyrus and left intraparietal sulcus). Again, more activity was found in parietal than in prefrontal areas during shifting tasks. In agreement with the meta-analysis of Wager et al. (2004) and with neuropsychological studies (Gehring \& Knight, 2002), these data suggest that parietal areas play a more basic functional role in shifting processes than prefrontal areas or, in other words, that parietal areas underlie cognitive processes that are less strategic or less linked to individual strategies than those underlie by prefrontal regions. Finally, only weak common activity was found for the inhibitory tasks, while interaction analyses demonstrated some specificity in the right orbitofrontal gyrus and the right middle/superior frontal gyrus. Again, these results support those of previous inhibitory studies using subtraction design paradigms: regions evidenced in the interaction analyses were frequently found to be activated in studies using the Stroop task (Bench et al., 1993; Bush et al., 1998; George et al., 1994; Larrue et al., 1994; Pardo et al., 1990; Taylor et al., 1997), and Aron et al. (2004) proposed that the right inferior frontal region is involved in the suppression of irrelevant responses.

The most interesting data obtained in this study concern cerebral areas that are common to the three executive processes. Previous studies had suggested that executive functioning is based on a network of anterior and posterior cerebral areas and is not subserved by the frontal lobes alone. Our results are 
Published in : Neuroscience (2006), 139 (1), 209-221

Status : Postprint (Author's version)

compatible with the conceptualisation of executive functioning in terms of interrelationships between anterior and posterior cerebral areas (Collette \& Van der Linden, 2002; D’Esposito \& Grossman, 1996; Fuster, 1993; Morris, 1994; Weinberger, 1993). Moreover, the results obtained in this study emphasise the critical role of the parietal areas in executive functioning, since the left superior parietal cortex and the right intraparietal sulcus are activated by all three executive processes in each and every subject. To date, very few neuroimaging data clearly attribute a role to these areas in connection with executive functioning and there has been no general agreement concerning their individual function. On the other hand, although the lateral frontal gyrus has frequently been associated with executive functioning, a wide range of functions have been attributed to this area: manipulation of information (Collette et al., 1999; D'Esposito et al., 1999; Postle et al., 1999), dual task coordination (D’Esposito et al., 1995, shifting processes (Kübler et al., 2003; Nagahama et al., 2001; Rogers et al., 2000), and inhibition (Chee et al., 2000; Collette et al., 2001). On the basis of a behavioural study using latent variable analyses, Miyake et al. (2000) proposed that cognitive processes common to updating, shifting and inhibition could relate to either the maintenance of goal and context information concerning the task to be performed or to inhibitory abilities. In agreement with Miyake et al.'s cognitive proposal, we suggested that the right intraparietal sulcus is involved in selective attention to behaviourally relevant stimuli and suppression of task-irrelevant information (Wojciulik \& Kanwisher, 1999). The left superior parietal cortex could be involved in establishing an attentional set to maintain or actively suppress working-memory representations during the accomplishment of executive tasks (Corbetta \& Shulman, 2002). Finally, the systematic activation of the middle and inferior lateral prefrontal cortex by a wide range of executive tasks suggests that they are involved in general executive processes. Recently, Smith et al. (2002; Wager \& Smith, 2003) proposed that one function of the dorsolateral prefrontal cortex could be temporal coding of representations to be processed. Indeed, all tasks that require the reordering of a temporal sequence or maintaining memory for temporal order routinely activate this region (for a similar interpretation, see also Fuster, 2001; Koechlin et al., 2003). Consequently, the prefrontal cortex would be involved in the temporal organisation necessary to the selection and initiation of the function to be performed in accordance with the task rules and goals, while parietal areas would be involved, as discussed previously, in the establishment of attentional sets responsible for the reactivation and suppression of working-memory contents necessary to perform the executive task (Kübler et al., 2003). This is generally consistent with Miyake et al.'s (2000) suggestion that commonalities between their nine executive tasks could be due to the selection and control of goal and context information concerning the current task.

\subsubsection{Limitations on the conjunction activation studies}

There are still some limits on the use of tasks associated with cognitive factors identified on the basis of variable latent analyses to explore the neural substrates of executive functioning. Indeed, the use of this kind of procedure depends on strong theoretical hypotheses about the structure of the factors whose neural substrates are explored. At present, theoretical models of executive functioning are not well enough specified at a cognitive level to easily allow the conceptualisation of such working hypotheses. Moreover, the model obtained with this method remains 'constrained' by the choice of tasks, and the possibility cannot be ruled out that a change in the task battery would modify the structure of the factors obtained. Finally, it is not certain that there is a strict correspondence between the factors identified at a cognitive level and changes in regional cerebral blood flow. For example, the running of a specific executive process might be expressed by the synchronisation of cerebral activation between several cerebral areas and not by an increase in cerebral activity in one specific brain region.

Thus, in order to obtain a better representation of the cerebral areas underlying executive functioning, it is important to explore the relationships between the data obtained using various experimental designs. Although conjunction analyses highlight common cerebral areas involved in the accomplishment of different tasks, it seems essential to construct more specific experimental designs that use subtraction and interaction analyses or explore effective and functional connectivity, in order to better understand the functional role of these regions (for a detailed discussion of the advantages and limitations of these statistical analyses, see Friston \& Price, 2001; Price et al., 1997). Another way of better 
Published in : Neuroscience (2006), 139 (1), 209-221

Status : Postprint (Author's version)

understanding the neural substrates of executive functioning consists of exploring with fMRI the temporal dynamics of cerebral areas associated with specific executive tasks. This point will be tackled in the next section.

\section{The temporal dynamic of activations in executive functioning}

Although the neural substrates of executive functioning are relatively well understood, very few studies have been interested in demonstrating the functional role of these areas by exploring the presence of sustained or transient cerebral activity, these two kinds of temporal dynamics being respectively attributed to state- and item-related processes in episodic memory (i.e., Otten et al., 2002). So far, three fMRI studies have simultaneously explored sustained and transient activity in the domain of executive functioning, using an n-back task (Cohen et al., 1997), a running span task (Collette et al., 2005b) and a shifting task (Braver et al., 2003).

Cohen et al. (1997) used $n$-back tasks with various memory loads (0-back, 1-back, 2-back, 3back). The authors hypothesised that areas involved in working memory would vary as a function of memory load, with greater activation at higher load levels. Furthermore, regions involved in active maintenance would exhibit sustained activation throughout the trial, whereas those involved in timelimited functions (such as updating) would peak higher (or last longer) at higher levels of load. Continuous activity was found in the dorsolateral prefrontal cortex (BA 46/9), Broca's area and the posterior parietal cortex. Transient activity was found in Broca's area (a region adjacent to that described as presenting continuous activity) and the supramarginal gyrus. Activity in Broca's area was explained in terms of subvocal rehearsal of verbal material rather than updating. Surprisingly, no transient cerebral activity was found in the dorsolateral prefrontal cortex and the authors suggested that this region played a role in the active maintenance of information in working memory but not in the updating of memory contents, as previously stated.

The pattern of temporal dynamics of the neural substrates associated with updating was recently re-examined using a running span task, which allows for better control over the storage aspects of the task (Collette et al., 2005b). Sustained activity specifically linked to updating was found in the left precentral cortex, the inferior (BA 47) and middle (BA 46) frontal gyrus bilaterally, the left inferior and posterior frontal gyrus (BA 44/45), the frontopolar (BA 10) cortex bilaterally, the medial frontal gyrus (BA 9) and the anterior cingulate (BA 32). Increases in cerebral activity associated with posterior cerebral areas were located in the left superior parietal (BA 7) and intraparietal sulcus. Transient cerebral activity associated to the presentation of items to update was located in the ventral part of the medial frontal gyrus (BA 11). Among the network of areas demonstrating sustained activity, the left superior parietal region and the frontopolar cortex were associated to specific updating processes, respectively an ordination process (Marshuetz et al., 2000; Postle et al., 2001) and the evaluation of internally generated information (Christoff \& Gabrielli, 2000), while transient activity in the medial frontal gyrus was associated with inhibitory of information that becomes irrelevant in working memory (Collette et al., 2005b; for similar data on episodic memory, see Schnider et al., 2000).

Finally, Braver et al. (2003) explored neural mechanisms of transient and sustained cognitive control during task switching. Indeed, the performance costs of task switching can be decomposed into trial-specific effects (switching costs) and non-specific effects (mixing cost). Switching costs were evaluated by contrasting, in the same block, trials in which the task was simply repeated (termed taskrepeat trials) and those in which the task was shifted (termed task-switch trials). Mixing cost can be isolated by comparing performance on task-repeat trials in blocks where a single task was performed (single-task blocks) against performance in blocks where subjects have to alternate between repeat and switch tasks (mixed-task blocks). Braver et al. alternated blocks of single tasks and mixed tasks. Within each block, activity was decomposed into that which was event-related (i.e., due to the performance of the trial) and that which was state-related (i.e., due to increased activity for the block). Brain regions that showed sensitivity to transient aspects of the shifting process were identified via the event-related contrast 
Published in : Neuroscience (2006), 139 (1), 209-221

Status : Postprint (Author's version)

of task switch to task repeat. These regions were the left dorsolateral prefrontal cortex (BA 44/9), the left ventrolateral prefrontal cortex (BA 45/47) and the left superior parietal. Brain regions sensitive to sustained aspects of shifting were the right ventral anterior cingulate (BA 24), the right medial anterior prefrontal cortex (BA 9/10), and the right lateral anterior prefrontal cortex (BA 46/10). These regions were identified via the state-related contrast of mixed tasks and single tasks. Sustained activation of the right anterior prefrontal cortex was attributed to the maintaining of a heightened level of cognitive control over an extended period in situations requiring rapid and flexible alternation between multiple different tasks. Transient activity in the lateral prefrontal cortex may reflect the internal representation and maintenance of task-set information. Finally, transient superior parietal cortex activation might reflect processes associated with the online reconfiguration and updating of task-set information immediately following a switch in the task.

Taken as a whole, these three studies demonstrate that the neural substrates of executive functioning can also be characterised by the presence of both sustained and transient cerebral activity. Given the small number of studies that tackled this question with regard to executive functioning, as well as the methodological differences between these studies, no firm conclusions about the functional role of these regions can be drawn at present. Nevertheless, in a given executive task, sustained activity can be related to general cognitive processes (such as maintaining the current state of attention or cognitive control) as well to more specific executive processes (for example, the ordination process in updating). Specific executive processes were also found to be associated with transient activation. So the suppression of information that becomes irrelevant was associated with transient activation in the ventral part of the medial prefrontal cortex during the updating task (Collette et al., 2005b), and transient activity in the lateral prefrontal cortex may be related to the maintenance of task-set information during the shifting task (Braver et al., 2003). Finally, it must be emphasised that different cerebral areas with sustained and transient activity were observed in the studies by Cohen et al. (1997) and Collette et al. (2005b), both of which explored the updating process (i.e., transient activity was respectively in Broca's area and in the medial frontal gyrus). Although the reasons for these discrepancies are not clear at this time, we recently obtained PET data indicating that three updating tasks varying according to the material (words, consonants or sounds) and procedure (recall or recognition) involve different cerebral networks, even if the three tasks have some cerebral areas in common (Collette et al., 2005c). Such a distinction could also exist at the level of the temporal dynamics of cerebral areas. However, further studies will necessary for a better comprehension of these dynamic aspects.

\section{Conclusions}

The exploration of executive functioning using functional neuroimaging techniques is a welldeveloped field of research. However, given the characteristics of executive processes, it is quite difficult to determine their neural substrates. Consequently, the use of task-specific paradigms demonstrated a great heterogeneity in the cerebral areas involved during the performance of executive tasks. This heterogeneity was apparent not only between tasks that assessed different processes but also between tasks that were supposed to explore the same process (e.g., dual-task coordination). However, these studies clearly demonstrated that the different executive functions are associated with both prefrontal and posterior (mainly parietal) regions. Conjunction analyses confirm these findings. Moreover, conjunction analyses allow better control over the multi-compound aspects of executive tasks and the difficulty to find wellmatched control tasks. These analysis emphasised the role of posterior areas in executive functioning. They also demonstrate that the evidence of specificity and commonality of executive processes found by Miyake et al. (2000) at the cognitive level also existed at the cerebral level. Finally, recent studies taking into account the temporal dynamics of the cerebral areas involved in executive tasks have demonstrated that regions associated with executive processes can be characterised by continuous or transient cerebral activity, which presupposes that these areas subserve different functional roles. 
Published in : Neuroscience (2006), 139 (1), 209-221

Status : Postprint (Author's version)

However, the considerable heterogeneity of findings highlights the methodological limitations of functional neuroimaging to explore highly-integrated processes such as executive functioning. So, we consider that more progress in understanding the neural substrates of these processes will be achieved only if data obtained from the fields of cognitive psychology, neuropsychology, electrophysiology and functional neuroimaging are taken into account simultaneously in an integrated way. The contribution of cognitive psychology should concern not only a better comprehension of the interrelationships (i.e., diversity and commonality) of the different executive processes but also a better understanding of the basic processes involved in executive tasks. For example, the process identified by Miyake et al. (2000) as updating seems to be composed of a series of subprocesses such as serial order processing, inhibition, and so on. Furthermore, the identification of changes in cerebral activity in normal subjects during executive tasks only supports inferences about the engagement of a particular brain system by that process, but does not entail that the system is necessary for this process. The administration of such tasks to patients with well-circumscribed frontal or parietal brain lesions should allow one to dissociate those cerebral areas that are essential to the performance of the task from the other cerebral areas activated in normal subjects. Similarly, the use of transcranial magnetic stimulation in normal subjects should allow us to simulate brain lesions in cerebral areas previously associated with the performance of an executive task. The observation of performance and patterns of errors following magnetic stimulation should also make it possible to better determine the functional role of this region. 
Published in : Neuroscience (2006), 139 (1), 209-221

Status : Postprint (Author's version)

References

Adcock RA, Constable RT, Gore JC, Goldman-Rakic PS (2000) Functional neuroanatomy of executive processes involved in dual task performance. Proc Nat Acad Sci USA 97:3567-3572.

Ahola K, Vilkki J, Servo A (1996) Frontal tests do not detect frontal infarctions after ruptured intracranial aneurysm. Brain Cogn 31:1-16.

Andrès P, Van der Linden M (1998) Les capacités d'inhibition: une fonction frontale? Eur Rev Applied Psychol 48:33-38.

Andrès P, Van der Linden M (2000) Age-related differences in supervisory attentional system functions. J Gerontol B Psychol Sci 55:373-380.

Andrès P, Van der Linden M (2001) Supervisory Attentional System in patients with focal frontal lesions. J Clin Exp Neuropsychol 23:225-239.

Aron AR, Robbins TW, Poldrack RA (2004) Inhibition and the right inferior frontal cortex. Tr Cog Sci 8:170-177.

Awh E, Jonides J, Smith EE, Schumacher EH, Koeppe RA, Katz S (1996) Dissociation of storage and rehearsal in verbal working memory: Evidence from positron emission tomography. Psychol Sci 7:25-31.

Baddeley AD (1986) Working memory. Oxford: Clarendon Press.

Baddeley AD (1996) Exploring the central executive. Q J Exp Psychol A 49:5-28.

Baddeley AD, Della Sala S, Papagno C, Spinnler H (1997) Dual-task performance in dysexecutive and nondysexecutive patients with a frontal lesion. Neuropsychology 11:187-194.

Baker SC, Rogers RD, Owen AM, Frith CD, Dolan JR, Frackowiak RSJ, Robbins TW (1996) Neural systems engaged by planning: a PET study of the Tower of London task. Neuropsychologia 34:515-526.

Bench CJ, Frith CD, Grasby PM, Friston KJ, Paulesu E, Frackowiak RSJ, Dolan RJ (1993) Investigations of the functional anatomy of attention using the Stroop test. Neuropsychologia 31:907-922.

Berman KF, Ostrem JL, Randolph C, Gold JM, Goldberg TE, Coppola R, Carson RE, Herscovitch P, Weinberger DR (1995) Physiological activation of a cortical network during performance of the Wisconsin Card Sorting Test. Neuropsychologia 33:1027-1046.

Braver TS, Cohen JD, Nystrom LE, Jonides J, Smith EE, Noll DC (1997) A parametric study of prefrontal cortex involvement in human working memory. Neuroimage 5:49-62.

Braver TS, Reynolds JR, Donaldson DI (2003). Neural mechanisms of transient and sustained cognitive control during task switching. Neuron 39: 713-726.

Bunge SA, Klingberg T, Jacobsen RB, Gabrieli JDE (2000) A resource model of the neural basis of executive working memory. Proc Nat Acad Sci USA 97:3573-3578.

Burgess PW (1997) Theory and methodology in executive function research. In: Methodology of frontal and executive functions (Rabbit P, ed), pp 81-116 Hove: Psychology Press. 
Published in : Neuroscience (2006), 139 (1), 209-221

Status : Postprint (Author's version)

Burgess PW, Alderman N, Evans J, Emslie H, Wilson BA (1998) The ecological validity of tests of executive function. J Int Neuropsychol Soc 4:547-558.

Burgess PW, Shallice T (1994) Fractionnement du syndrome frontal. Revue de Neuropsychologie 4:345370.

Burgess PW, Shallice T (1996a) Bizarre responses, rule detection and frontal lobe lesions. Cortex. 32: 241-259.

Burgess PW, Shallice T (1996b) Response suppression, initiation and strategy use following frontal lobe lesions. Neuropsychologia 34:263-273.

Bush G, Whalen PJ, Rosen BR, Jenike MA , McInerney SC, Rauch SL (1998) The counting Stroop: An interference task specialized for functional neuroimaging. Validation study with functional MRI. Hum Brain Mapp 6:270-282.

Chee MWL, Sriram N, Siong Soon C, Ming Lee K (2000) Dorsolateral prefrontal cortex and the implicit association of concepts and attributes. Neuroreport 11:135-140.

Christoff K, Gabrielli JDE (2000) The frontopolar cortex and human cognition: Evidence for a rostrocaudal hierarchical organization within the human prefrontal cortex. Psychobiol 28:168-186.

Cohen JD, Perlstein WM, Braver TS, Nystrom LE, Noll DC, Jonides J, Smith EE (1997) Temporal dynamics of brain activation during a working memory task. Nature 386:604-607.

Collette F, Olivier L, Van der Linden M, Laureys S, Delfiore G, Luxen A, Salmon E (2005a). Involvement of both prefrontal and inferior parietal cortex in dual task performance. Cog Brain Res (In press).

Collette F, Salmon E, Balteau E, Degueldre C, Luxen A, Maquet P. (2005b). Updating information in working memory elicits both transient and sustained brain responses. Submitted.

Collette F, Salmon E, Van der Linden M, Chicherio C, Belleville S, Degueldre C, Delfiore G, Franck G (1999) Regional brain activity during tasks devoted to the central executive of working memory. Cog Brain Res 7:411-417.

Collette F, Van der Linden M (2002) Brain imaging of the central executive component of working memory. Neurosci Biobehav Rev 26:105-125.

Collette F, Van der Linden M, Delfiore G, Degueldre C, Luxen A, Salmon E (2001) The functional anatomy of inhibition processes investigated with the Hayling task. Neuroimage. 14:258-267.

Collette F, Van der Linden M, Laureys S, Delfiore G, Degueldre C, Luxen A, Salmon E (2005c). Exploring the unity and diversity of the neural substrates of executive functioning. Hum Brain Mapp DOI 10/1002/hbm.20118.

Corbetta M, Shulman GL (2002) Control of goal-directed and stimulus-driven attention in the brain. Nat Rev 31:201-215.

Cowey CM, Green S (1996) The hippocampus: A "working memory" structure? The effect of hippocampal sclerosis on working memory. Memory 4:19-30. 
Published in : Neuroscience (2006), 139 (1), 209-221

Status : Postprint (Author's version)

Curtis CE, D’Esposito M (2003) Persistent activity in the prefrontal cortex during working memory. Trends Cog Sci 7: 415-423.

Dagher M, Owen AM, Boecker H, Brooks D (1999). Mapping the network for planning: a correlational PET activation study with the Tower of London task. Brain 122: 1973-1987.

Dempster FN, Corkill AJ (1999) Individual differences in susceptibility to interference and general cognitive ability. Acta Psychol 101:395-416.

D'Esposito M, Detre JA, Alsop CD, Shin RK, Atlas S, Grossman M (1995) The neural basis of the central executive of working memory. Nature 378:279-281.

D'Esposito M, Postle BR, Ballard D, Lease J (1999) Maintenance versus manipulation of information held in working memory: An event-related fMRI study. Brain Cogn 41:66-86.

D'Esposito M, Postle BR, Jonides J, Smith EE (1999) The neural substrate and temporal dynamics of interference effects in working memory as revealed by event-related functional MRI. Proc Nat Acad Sci USA 96:7514-7519.

D'Esposito M., Grossman M (1996) The physiological basis of executive function and working memory. The Neuroscientist 2:345-352.

Duncan J (1995) Attention, intelligence and the frontal lobes. In: The cognitive neurosciences (Gazzaniga MS, ed), pp 721-731 Cambridge, MA: MIT Press.

Duncan J, Johnson R, Swales M, Freer C (1997) Frontal lobe deficits after head injury: Unity and diversity of function. Cog Neuropsychol 14:713-741.

Duncan J, Owen AM (2000) Common regions of the human frontal lobe recruited by diverse cognitive demands. Tr Neurosci 23:475-483.

Engle RW, Kane MJ, Rettinger DA (1999) Individual differences in working memory capacity and what they tell us about controlled attention, general fluid intelligence, and functions of the prefrontal cortex. In: Models of working memory: Mechanisms of active maintenance and executive control (Miyake A, Shah P, eds), pp 102-134 New-York: Cambridge University Press.

Fink GR, Halligan PW, Marshall JC, Frith CD, Frackowiak RSJ, Dolan RJ (1997) Neural mechanisms involved in the processing of global and local aspects of hierarchically organized visual stimuli. Brain 120:1779-1791.

Friedman NP, Miyake A (2004) The relations among inhibition and interference control functions: A latent-variable analysis. J Exp Psychol Gen 133:101-135.

Friston KJ, Frith CD, Fletcher P, Liddle PF, Frackowiak RSJ (1996) Functional topography: Multidimensional scaling and functional connectivity in the brain. Cereb Cortex 6:156-164.

Friston KJ, Price CJ (2001) Generative models, brain function and neuroimaging. Scand J Psychol 42:167177.

Frith CD, Friston KJ, Liddle PF, Frackowiak RSJ (1991). A PET study of word finding. Neuropsychologia, 29: 1137-1148. 
Published in : Neuroscience (2006), 139 (1), 209-221

Status : Postprint (Author's version)

Fuster JM (2001) The prefrontal cortex - An update: Time is of the essence. Neuron 30:319-333.

Fuster JM (1993) Frontal lobes. [Review]. Curr Opin Neurobiol 3:160-165.

Garavan H, Ross TJ, Li SJ, Stein EA (2000) A parametric manipulation of central executive functioning. Cereb Cortex 10:585-92.

Garavan H, Stein EA (1999) Right hemispheric dominance of inhibitory control: An event-related functional MRI study. Proc Nat Acad Sci USA 96:8301-8306.

Gehring WJ, Knight RT (2002) Lateral prefrontal damage affects processing selection but not attention switching. Cogn Brain Res 13:267-279.

George MS, Ketter TA, Parekh PI, Rosinsky N, Ring H, Casey BJ, Trimble MR, Horwitz B, Herscovitch P, Post RM (1994) Regional brain activity when selecting a response despite interference: a H2O15 PET study of the Stroop and emotional Stroop. Hum Brain Mapp 1:194-209.

Gurd JM, Amunts K, Weiss PH, Zafiris O, Zilles K, Marshall JC, Fink GR (2002) Posterior parietal cortex is implicated in continuous switching between verbal fluency tasks: an fMRI study with clinical implications. Brain 125:1024-1038.

Herath P, Klingberg T, Young J, Amunts K, Roland P (2001) Neural correlates of dual task interference can be dissociated from those of divided attention: an fMRI study. Cereb Cortex 11:796-805.

Jahanshashi M, Dirnberger G, Fuller R, Frith CD (2000) The role of the dorsolateral prefrontal cortex in random number generation: A study with positron emission tomography. Neuroimage 12:713-725.

Jonides J, Schumacher EH, Smith EE, Lauber EJ, Awh E, Minoshima S, Koeppe RA (1997) Verbal working memory load affects regional brain activation as measured by PET. J Cogn Neurosci 9:462-475.

Jonides J, Smith EE, Marshuetz C, Koeppe RA, Reuter-Lorenz PA (1998) Inhibition in verbal working memory revealed by brain activation. Proc Nat Acad Sci USA 95:8410-8413.

Klingberg T (1998) Concurrent performance of two working memory tasks: Potential mechanisms of interference. Cereb Cortex 8:593-601.

Koechlin E, Ody C, Kouneiher F (2003) The architecture of cognitive control in the human prefrontal cortex. Science 302:1181-1185.

Kübler A, Murphy K, Kaufman J, Stein EA, Garavan H (2003) Co-ordination within and between verbal and visuospatial working memory: network modulation and anterior frontal recruitment. Neuroimage 20:1298-308.

Larrue V, Celsis P, Bès A, Marc-Vergnes JP (1994) The functional anatomy of attention in humans: Cerebral blood flow changes induced by reading, naming and the Stroop effect. J Cereb Blood Flow Metab 14:958-962.

Letho J (1996) Are executive function tests dependent on working memory capacity? Q J Exp Psychol A 49:29-50. 
Published in : Neuroscience (2006), 139 (1), 209-221

Status : Postprint (Author's version)

Logan GD (1994) On the ability to inhibit thought and action: A user's guide to the stop-signal paradigm. In: Inhibition processes in attention, memory and language (Dagenbach D, Carr TH, eds), pp 189-239 San Diego, CA: Academic press.

Lowe C, Rabbitt P (1997) Cognitive models of aging and frontal lobe deficits. In: Methodology of frontal and executive functions (Rabbitt P, ed), pp 39-59 Hove, UK: Psychology Press.

Marshuetz C, Smith EE, Jonides J, DeGutis J, Chenevert TL (2000). Order information in working memory: fMRI evidence for parietal and prefrontal mechanisms. J Cogn Neurosci 12 (suppl 2): 130-144.

Miyake A, Friedman NP, Emerson MJ, Witzki AH, Howerter A (2000) The unity and diversity of executive functions and their contribution to complex "frontal lobe" tasks: A latent variable analysis. Cognit Psychol 41:49-100.

Morris N, Jones DM (1990) Memory updating in working memory: The role of the central executive. Br J Psychol 81:111-121.

Morris RG (1994) Recent developments in the neuropsychology of dementia. Int Rev Psych 6:85-107.

Morris RG, Ahmed S, Syed GM, Toone BK (1993). Neural correlates of planning ability: Frontal lobe activation during the Tower of London test. Neuropsychologia, 31: 1367-1378.

Mountain MA, Snow-William WG (1993) Wisconsin Card Sorting Test as a measure of frontal lobe pathology: A review. Clin Neuropsychol 7:108-118.

Nagahama Y, Fukuyama H, Yamauchi H, Matsuzaki S, Konishi J, Shibasaki H, Kimura J (1996) Cerebral activation during performance of a card sorting test. Brain 119:1667-1675.

Nagahama Y, Okada T, Katsumi Y, Hayashi T, Yamauchi H, Oyanagi C, Konishi J, Fukuyama H, Shibasaki H (2001): Dissociable mechanisms of attentional control within the human prefrontal cortex. Cereb Cortex 11: 85-92.

Navon D (1977) Forest before trees: The precedence of global features in visual perception. Cognit Psychol 9:353-383.

Nelson JK, Reuter-Lorenz PA, Sylvester C-Y C, Jonides J., Smith, EE (2003). Dissociable neural mechanisms underlying response-based and familiarity-based conflict in working memory. Proc Nat Acad Sci USA 100: 11171-11175.

Newman SD, Carpenter PA, Varma S, Just MA (2003). Frontal and parietal participation in problem solving in the Tower of London: fMRI and computational modelling of planning and high-level perception. Neuropsychologia 41: 1668-1682.

Nigg JT (2000) On inhibition/disinhibition in developmental psychopathology: Views from cognitive and personality psychology and a working inhibition taxonomy. Psychol Bull 126:220-246.

Norman DA, Shallice T (1986) Attention to action: Willed and automatic control of behavior. In: Consciousness and self regulation. Advances in research and theory (Davidson RJ, Schwartz GE, Shapiro D, eds), pp 1-18 New-York: Plenum Press.

Otten LJ, Henson RNA, Rugg MD (2002) State-related and item-related neural correlates of successful memory encoding. Nat Neurosci 5: 1339-1344. 
Published in : Neuroscience (2006), 139 (1), 209-221

Status : Postprint (Author's version)

Owen AM, Stern CE, Look RB,Tracey I, Rosen BR, Petrides M (1998) Functional organization of spatial and nonspatial working memory processing within the human lateral frontal cortex. Proc Nat Acad Sci USA 95 : 7721-7726

Owen AM, Downes JD, Sahakian BJ, Polkey CE, Robbins TW (1990).Planning and spatial working memory following frontal lobe lesions in man. Neuropsychologia 28: 1021-1034.

Pardo JV, Pardo PJ, Janer KW, Raichle ME (1990) The anterior cingulate cortex mediates processing selection in the Stroop attentional conflict paradigm. Proc Nat Acad Sci USA 87:256-259.

Paulesu E, Frith CD, Frackowiak RSJ (1993) The neural correlates of the verbal component of working memory. Nature 362:342-345.

Paulesu E, Goldacre B, Scifo P, Cappa SF, Giraldi MC, Castiglioni I, Perani D, Fazio F (1997) Functional heterogeneity of left inferior frontal cortex as revealed by fMRI. Neuroreport 8:2011-2016.

Perret E (1974) The left frontal lobe of man and the suppression of habitual responses in verbal categorical behavior. Neuropsychologia 12:323-330.

Phelps EA, Hyder F, Blamire AM, Shulman, RG (1997) FMRI of the prefrontal cortex during overt verbal fluency. Neuroreport 8: 561-565.

Pollack I, Johnson LB, Knaff PR (1959) Running memory span. J Exp Psychol 57: 137-146.

Pochon JB, Levy R, Poline JB, Crozier S, Lehericy S, Pillon B, Deweer B, Le Bihan D, Dubois B (2001) The role of dorsolateral prefrontal cortex in the preparation of forthcoming actions: an fMRI study.Cereb Cortex 11:260-6.

Postle B, Berger JS, Goldstein JH, Curtis CE, D’Esposito M (2001). Behavioral and neuropsychological correlates of episodic coding, proactive interference, and list length effects in a running span verbal working memory task. Cogn Affect Behav Neurosci 1: 10-21.

Postle BR, Berger JS, D'Esposito M (1999) Functional neuroanatomical double dissociation of mnemonic and executive control processes contributing to working memory performance. Proc Nat Acad Sci USA 96:12959-12964.

Price CJ, Moore CJ, Friston KJ (1997) Subtractions, conjunctions and interactions in experimental design of activation studies. Hum Brain Mapp 5:264-272.

Rabbit P (1997) Introduction: Methodologies and models in the study of executive function. In: Methodology of frontal and executive function (Rabbit P, ed), pp 1-38 Hove: Psychology Press.

Ragland JD, Glahn DC, Gur DC, Censits DM, Smith, RJ, Mozley PD, Alavi A, Gur RE (1997). PET regional cerebral blood flow change during working and declarative memory: Relationship with task performance. Neuropsychology, $11: 222-231$.

Robbins TW, Owen AM, Sahakian BJ, Lawrence AD, McInnes L, Rabbitt PMA (1998) A study of performance on tests from the CANTAB battery sensitive to frontal lobe dysfunction in a large sample of normal volunteers: Implications for theories of executive functioning and cognitive aging. $\mathrm{J}$ Int Neuropsychol Soc 4:474-790. 
Published in : Neuroscience (2006), 139 (1), 209-221

Status : Postprint (Author's version)

Roberts RJ, Hager LD, Heron C (1994) Prefrontal cognitive processes: Working memory and inhibition in the anti-saccade task. J Exp Psychol Gen 123:374-393.

Rogers RD, Andrews TC, Grasby PM, Brooks DJ, Robbins TW (2000) Contrasting cortical and subcortical activations produced by attentional-set shifting and reversal learning in humans. J Cogn Neurosci 12:142-162.

Salmon E, Van der Linden M, Collette F, Delfiore G, Maquet P, Degueldre C, Luxen A, Franck G (1996) Regional brain activity during working memory tasks. Brain 119:1617-1625.

Schnider A, Treyer V, Buck A (2000). Selection of currently relevant memories by the human posterior medial orbitofrontal cortex. The J Neurosci 20: 5880-5884.

Schumacher EH, Lauber E, Awh E, Jonides J, Smith EE, Koeppe RA (1996) PET evidence for an amodal verbal working memory system. Neuroimage 3:79-88.

Shallice T (1982) Specific impairments of planning. Philos Trans R Soc Lond B Biol Sci 298:199-209.

Shallice T, Burgess P (1991) Higher-order cognitive impairments and frontal lobe lesions in man. In: Frontal lobe functions and dysfunctions (Levin HS, Eisenberg HM, Benton AL, eds), pp 125-137 NewYork: Oxford University Press.

Shallice T, Burgess PW (1993) Supervisory control of action and though selection. In: Attention: selection, awareness and control: A tribute to Donald Broadbent (Baddeley AD, Weiskrantz L, eds), pp 171-187 Oxford: Oxford University Press.

Shimamura AP (2000) The role of prefrontal cortex in dynamic filtering. Psychobiol 28:207-218.

Sidtis JJ, Strother SC, Anderson JR, Rottenberg DA (1999) Are brain functions really additive? Neuroimage 6:490-496.

Simkins-Bullock J, Brown GG, Greiffenstein M, Malik GM, McGillicuddy J (1994) Neuropsychological correlates of short-term memory distractor tasks among patients with surgical repair of anterior communicating artery aneurysms. Neuropsychology 8:246-254.

Smith EE, Geva A, Jonides J, Miller A, Reuter-Lorenz PA, Koeppe RA (2001) The neural basis of task switching in working memory: Effect of performance and aging. Proc Nat Acad Sci USA 98:2095-2100.

Smith EE, Jonides J, Koeppe RA (1996) Dissociating verbal and spatial working memory using PET. Cereb Cortex 6:11-20.

Smith EE, Marshuetz C, Geva A (2002) Working memory: findings from neuroimaging and patient studies. In: Handbook of Neuropsychology, $2^{\text {nd }}$ edition (Grafman J, ed), pp 55-72 Amsterdam: Elsevier.

Sohn M, Ursu S, Anderson JR, Stenger VA, Carter CS (2000) The role of prefrontal cortex and posterior parietal cortex in task switching. Proc Nat Acad Sci 97:13448-13453.

Stroop JR (1935) Studies of interference in serial verbal reactions. J Exp Psychol 6:643-661.

Taylor SF, Kornblum S, Lauber EJ, Minoshima S, Koeppe RA (1997) Isolation of specific interference processing in the Stroop task: PET activation studies. Neuroimage 6:81-92. 
Published in : Neuroscience (2006), 139 (1), 209-221

Status : Postprint (Author's version)

Tipper SP (1991) Less attentional selectivity as a result of declining inhibition in older adults. Bull. Psychonom Soc 29:45-47.

Van der Linden M, Collette F, Salmon E, Delfiore G, Degueldre C, Luxen A, Franck G (1999) The neural correlates of updating of information in verbal working memory. Memory 7:549-560.

Vandenbergh R, Gitelman DR, Parrish TB, Mesulam MM (2001). Functional specificity of superior parietal mediation of spatial shifting. Neuroimage 14: 661-673.

Vilkki J, Virtanen S, Surma-Aho O, Servo A (1996) Dual task performance after focal cerebral lesions and closed head injuries. Neuropsychologia 34:1051-1056.

Wager TD, Jonides J, Reading S (2004) Neuroimaging studies of shifting attention: a meta-analysis. Neuroimage 22:1679-1693.

Wager TD, Smith EE (2003) Neuroimaging studies of working memory: A meta-analysis. Cogn Affect Behav Neurosci 3:255-274.

Weinberger DR (1993) A connectionist approach to the prefrontal cortex. J Neuropsychiatry Clin Neurosci 5:241-253.

Wilkinson DT, Halligan PW, Marshall JC, Büchel C, Dolan RJ (2001) Switching between the forest and the trees: Brain systems involved in local/global changed-level judgements. Neuroimage 13:56-67.

Wojciulik E, Kanwisher N (1999) The generality of parietal involvement in visual attention. Neuron 23:747-764.

Zacks RT, Hasher L, Radvansky G (1996) Studies of directed forgetting in older adults. J Exp Psychol Learn Mem Cogn 22:143-15. 